\title{
CXXXVI. THE DETERMINATION OF VITAMIN A WITH THE PHOTOELECTRIC COLORIMETER
}

\author{
BY WILLIAM JOHN DANN \\ Department of Physiology and Pharmacology, Duke University School of \\ Medicine, Durham, North Carolina \\ AND KENNETH AUSTIN EVELYN \\ McGill University Clinic, Royal Victoria Hospital, Montreal, Canada
}

(Received 21 March 1938)

THIs communication describes the application of the Evelyn photoelectric colorimeter [Evelyn, 1936] to the quantitative estimation of vitamin A by the antimony trichloride reaction of Carr \& Price [1926]. It was thought that the Carr-Price test with its attendant difficulties due chiefly to the instability of the colour to be measured (for data on this point, see below) might be performed more accurately with the photoelectric colorimeter on account of the rapidity with which readings can be taken on this instrument. The results described below show that the Carr-Price test can be performed with great precision by this method.

Apart from the biological assay there are two methods, one physical and the other chemical, which have been widely used for the estimation of vitamin A. The physical method is based on the discovery of Takahashi et al. [1925] that vitamin A concentrates show selective absorption in the ultraviolet spectral region with a maximum at $328 \mathrm{~m} \mu$. This method was introduced by Drummond \& Morton [1929], and its status as a fairly satisfactory method is based on critical examination by Coward et al. [1931], Morgan et al. [1935], the Medical Research Council [1935] and Hume [1937].

In the chemical method of Carr \& Price [1926], the vitamin A is made to react with antimony trichloride in chloroform solution, the amount of the resultant blue pigment being determined by examination of the solution in a tintometer or visual spectrophotometer. The use of the tintometer is attended by considerable error owing to the unavoidable difficulties in colour matching by eye, and also to the peculiar physical basis of tintometry which is responsible for the non-linear relation between the tintometer reading and the amount of vitamin A [Notevarp \& Weedon, 1936]. Spectrophotometric measurement shows, however, that the extinction coefficient of the blue solution at $620 \mathrm{~m} \mu$ is a linear function of the concentration of vitamin $\mathbf{A}$ in any given material [Notevarp \& Weedon, 1936]. That the chromogen which reacts with antimony trichloride to produce the blue colour is vitamin A itself is indicated by the observations of Holmes \& Corbet [1937] who found that a crystalline preparation of very high biological potency gave the characteristic reaction. In spite of the considerable inaccuracies inherent in methods available for measuring the intensity of the blue colour up to the present, it has been shown that the results of this chemical method are almost as closely correlated with the biological assay as are the results of the physical method of estimation [Morgan et al. 1935; cf. Table VI, p. 1654]. Thus the chemical and physical methods have equally sound foundations as shown by earlier observations. 
In the past the investigator has therefore had the choice of using the inaccurate tintometric chemical method requiring little capital outlay, or the more accurate spectrophotometric chemical or physical methods requiring expensive apparatus (and for the physical method consuming much time in the exposure, development and reading of the photographic plates). Recently a special photoelectric photometer utilizing the physical method has been described [McFarlan et al. 1937]. This instrument permits rapid measurement of the extinction coefficient at $328 \mathrm{~m} \mu$, but has the disadvantage that it cannot be applied to uses other than the estimation of vitamin $A$.

There is at present no evidence that measurement of absorption at $328 \mathrm{~m} \mu$ is any more closely related to biological activity than is the depth of the blue colour produced in the Carr-Price reaction, but it is generally agreed that the former quantity is more susceptible of accurate measurement than the latter as long as one is forced to use tintometers or visual spectrophotometers for examination of the blue colour. The advantages of the photoelectric colorimeter $^{1}$ [Evelyn, 1936] for the measurement of the very unstable blue colour of the Carr-Price reaction may be readily appreciated by the following brief description of the apparatus, which is essentially a single photocell direct-reading photoelectric photometer equipped with light filters. A beam of approximately monochromatic (filtered) light is directed upon a photoelectric cell connected to a galvanometer which records the intensity of the incident light directly on a scale of 100 divisions. An absorption cell containing the solution under test may be interposed between the lamp and the photocell, and the ratio of the final and initial galvanometer readings measures the light transmission of the coloured compound. Important features of the design of the apparatus, in addition to simplicity, rapidity and objectivity of operation, are extreme constancy of the light source, the use of filters of high selectivity and permanence of spectral characteristics, and strictly linear relation between galvanometer deflexion and light intensity. For the vitamin A determination it is also important that the colorimeter employs a test tube type of absorption cell the open end of which projects above the top of the apparatus, thus allowing reagents to be added to the test solution while the tube is actually in place in the path of the beam of light. This feature together with the direct reading nature of the device enables the operator to record at short intervals the development and fading of the colour for any period he desires beginning within a second or two after addition of the reagent, and makes it possible to measure the intensity of the blue colour at its true maximum which, as we shall show below, occurs immediately after addition of the reagent.

Another important component of the photoelectric colorimeter is the filter which is used to isolate a narrow spectral region in the vicinity of the region of maximum absorption of the solution under test. Since a complete set of highly selective filters has been developed as an integral part of the apparatus, one has no difficulty in selecting a filter (No. 620) to fulfil this requirement for the vitamin A-antimony trichloride complex. Fig. 1 shows spectrophotometric transmission curves of the blue compound and of the filter used in its determination.

1 The application of the Evelyn photoelectric colorimeter to the measurement of vitamin A was first suggested by Dr Charles May of the Children's Hospital, Boston, Massachusetts. Dr May has perfected a micro-method for the determination of vitamin A in serum, which is described in a paper now in the press.

The colorimeter including all the necessary filters can be obtained from the Rubicon Company, 29 North Sixth Street, Philadelphia, Pa., U.S.A. 
It should be emphasized at this point that the physical basis of photoelectric measurements made with spectral filters rests on an accurate mathematical foundation no less secure than that of conventional spectrophotometric determinations. The relation between the true spectrophotometric transmittance $T_{\text {A }}$

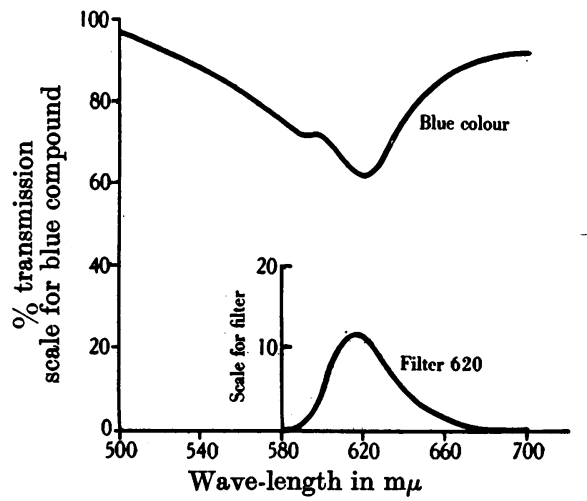

Fig. 1. Spectral characteristics of the vitamin A antimony trichloride blue colour and of the filter employed in its measurement.

at any wave-length $\lambda$, and the average transmittance $T_{\lambda}{ }^{\prime}$ which is measured with the corresponding filter is given by the equation

$$
T_{\lambda}{ }^{\prime}=\frac{\Sigma_{\lambda_{1}}^{\lambda_{2}} T_{\lambda} F_{\lambda} \cdot \delta \lambda}{\sum_{\lambda_{1}}^{\lambda_{2}} F_{\lambda} \cdot \delta \lambda},
$$

where $\lambda_{1}$ and $\lambda_{2}$ are the limits of transmission of the filter, and $F_{\lambda}$ is the transmittance of the filter at wave-length $\lambda$. These integrals can be evaluated rapidly and accurately from the known spectrophotometric curves of the coloured compound and filter by graphic representation and the use of the planimeter.

Finally it may be added that the filters are always made sufficiently selective to fulfil the requirement that the concentration of the coloured compound shall be proportional (within the limits of error of the determination itself) to the negative logarithm of $T^{\prime}$ as well as to that of $T$, thus allowing the use of standard spectrophotometric formulae in calculation of results obtained with the photoelectric colorimeter. To prevent confusion of terminology the following symbols are used throughout.

$$
\begin{aligned}
E & =-\log _{10} T \text { (spectrophotometer), } \\
L & =-\log _{10} T^{\prime} \text { (photoelectric colorimeter) } \\
& =-\log \frac{G}{100}=2-\log G,
\end{aligned}
$$

where $G$ is the galvanometer reading obtained on inserting the sample solution, the initial adjustment of the light intensity having been made to give a reading of 100 through a tube containing pure solvent only.

Procedure. The method is applicable to any fatty material containing vitamin A. If the vitamin A content of the material is small (as in cod liver oils) then the fat must be saponified and the unsaponifiable residue used for the test. This eliminates interference of unsaturated fatty acid radicals with the Carr-Price reaction [Emmerie, 1933]. It is suggested that saponification should be carried out whenever the vitamin A content of the material is believed to be less than 10,000 I.U. of vitamin A per g. 
A weighed amount of the fat or oil (about $250 \mathrm{mg}$. for a cod liver oil) is boiled in a small flask for $2 \mathrm{~min}$. with $2 \mathrm{ml}$. of $60 \%$ aqueous $\mathrm{KOH}$ and $10 \mathrm{ml}$. of $95 \%$ alcohol. The resulting solution is poured into $10 \mathrm{ml}$. of distilled water in a separating funnel, and the flask washed with two lots of $15 \mathrm{ml}$. of water and two lots of $25 \mathrm{ml}$. of ether, all being poured into the separating funnel. The funnel is shaken vigorously and the aqueous layer drawn off and discarded. The ethereal solution is washed vigorously with $10 \mathrm{ml}$. of water and then twice gently with $25 \mathrm{ml}$. of water each time and finally is dried by filtration through anhydrous sodium sulphate on a sintered glass diaphragm, and the sulphate washed with $20 \mathrm{ml}$. of ether. The ethereal solution is evaporated to dryness by connecting the containing flask directly to a water pump without using a condenser, the flask being immersed in warm water until the ether has evaporated, and then dipped for a moment in boiling water to drive off traces of water from the unsaponified residue.

The residue is dissolved in $25 \mathrm{ml}$. of chloroform, a suitable aliquot (say $0.25 \mathrm{ml}$. for first trial) is pipetted into one of the special photocolorimeter absorption test tubes, and the volume is made up to $1 \mathrm{ml}$. with chloroform. For a richer oil when saponification is unnecessary a few mg. should be weighed out and made up to a known volume with chloroform; a suitable aliquot of this solution is then transferred to the colorimeter tube. The appropriate filter (No. 620) is placed in the colorimeter, the apparatus is turned on, and the light intensity is adjusted so that a galvanometer deflexion of 100 divisions is obtained through a tube containing $1 \mathrm{ml}$. of chloroform and $9 \mathrm{ml}$. of reagent (or $10 \mathrm{ml}$. of reagent if more convenient, as it gives the same result). This blank tube is then replaced by the sample tube and $9 \mathrm{ml}$. of the antimony trichloride reagent (25 g. per $100 \mathrm{ml}$. of solution) are run rapidly down the side of the tube from an automatic pipette of the B.D.H. pattern or other suitable type ${ }^{1}$ of automatic pipette or burette.

As the reagent and test solution mix the galvanometer spot will move across the scale to a new position of temporary equilibrium which is recorded by the operator as $G_{620}$. If the reading is less than 20 or more than 70, another tube should be set up with a correspondingly smaller or larger volume of the chloroform solution.

The " $L$ value" of the oil may then be calculated from the equation

$$
L \text { value }=\frac{52.5}{M_{620}} \times L_{620},
$$

where $L_{620}=\left(2-\log G_{620}\right)$, and $M_{620} \mathrm{mg}$. is the weight of original material yielding the aliquot of unsaponifiable residue placed in the colorimeter tube.

Derivation of the new unit. The unit adopted for expression of results by the new method has been chosen to be as closely as possible analogous to the familiar $E(1 \%, 1 \mathrm{~cm} .620 \mathrm{~m} \mu)$ and is written $L(1 \%, 1 \mathrm{~cm} .620 \mathrm{~m} \mu)$. It is usually referred to as the " $L$ value". If $M \mathrm{mg}$. is the amount of original material corresponding to the volume of the chloroform solution used in the test, then the concentration of original material in the final blue solution is $M / 10 \mathrm{mg}$. per ml. or $M / 100 \mathrm{~g}$. per $100 \mathrm{ml}$. Bearing in mind that the effective thickness of the layer

1 The essential characteristic of whatever device is used for the delivery of the reagent solution is that its delivery time shall be no longer than 2 sec. The reading obtained is the same whether (a) the reagent is run into the tube already in position in the colorimeter, or $(b)$ the reagent is run into a tube held in the hand, the tube rapidly shaken and inserted into the colorimeter. This shows that complete mixing occurs rapidly when the reagent is run into the tube already in the colorimeter. 
of solution through which the light passes in the tubular photocolorimeter cell is $1.9 \mathrm{~cm}$. it is clear that the quantity which we have referred to above as $L_{620}$ is more exactly $L(M / 100 \%, 1.9 \mathrm{~cm} ., 620 \mathrm{~m} \mu)$ and therefore

$$
\begin{aligned}
L \text { value }=L(1 \%, 1 \mathrm{~cm} ., 620 \mathrm{~m} \mu) & =\frac{100}{M_{620}} \times \frac{1}{1 \cdot 9} \times L_{620} \\
& =\frac{52 \cdot 5}{M_{620}} \times L_{620} .
\end{aligned}
$$

By calculation from equation (1) it has been found that the ratio of $E$ (1\%, $1 \mathrm{~cm}$., $620 \mathrm{~m} \mu)$ to $L(1 \%, 1 \mathrm{~cm} ., 620 \mathrm{~m} \mu)$ is $1 \cdot 30 \pm 0.03$.

Correction for the presence of carotene. Since in the presence of antimony trichloride carotene develops a colour similar to that given by vitamin $\mathrm{A}$, a correction for carotene present should be applied to the $L$ value when the chloroform solution is seen to be yellow. To do this, $10 \mathrm{ml}$. of the solution are pipetted into another colorimeter tube, filter 620 is replaced by filter 440 , and after adjusting the galvanometer reading to 100 with a blank tube containing $10 \mathrm{ml}$. of chloroform the galvanometer reading with the tube of solution in place is recorded as $G_{440}$. If the reading is less than 20 , a suitable dilution of the solution is made to obtain a reading between 20 and 70 .

The "corrected $L$ value" of the oil may then be calculated from the equation

$$
L \text { value (corrected) }=52 \cdot 5\left(\frac{L_{620}}{M_{620}}-0 \cdot 14 \cdot \frac{L_{440}}{M_{440}}\right),
$$

where $L_{440}=\left(2-\log G_{440}\right)$, and $M_{440} \mathrm{mg}$. is the weight of original material yielding the aliquot of unsaponifiable residue contained in the tube used for recording $G_{440}$.

The observations which establish the constant $0 \cdot 14$ used in this equation are described below.

\section{Experimental}

Application of laws of Lambert and Beer to the blue solution, and precision of the method. It is clear that the definition of the new unit involves the assumption that $L$ (the negative logarithm of the transmittance) is a linear function of $M$

Table I. Data on the precision of estimation of vitamin $A$ with the photoelectric colorimeter. Readings of $L$ for different volumes of the same solution containing vitamin $A$.

\begin{tabular}{ccc}
$\begin{array}{c}\text { Volume of } \\
\text { solution (ml.) }\end{array}$ & \multicolumn{1}{c}{$L$} & $L \div$ volume \\
$1 \cdot 0$ & $0 \cdot 601$ & $0 \cdot 601$ \\
$0 \cdot 95$ & $0 \cdot 572$ & $0 \cdot 602$ \\
$0 \cdot 90$ & $0 \cdot 542$ & $0 \cdot 602$ \\
$0 \cdot 85$ & $0 \cdot 520$ & $0 \cdot 611$ \\
$0 \cdot 80$ & $0 \cdot 485$ & $0 \cdot 606$ \\
$0 \cdot 75$ & $0 \cdot 457$ & $0 \cdot 609$ \\
$0 \cdot 70$ & $0 \cdot 424$ & $0 \cdot 606$ \\
$0 \cdot 65$ & $0 \cdot 3955$ & $0 \cdot 608$ \\
$0 \cdot 60$ & $0 \cdot 365$ & $0 \cdot 608$ \\
$0 \cdot 55$ & $0 \cdot 3355$ & $0 \cdot 610$ \\
$0 \cdot 50$ & $0 \cdot 302$ & $0 \cdot 604$ \\
$0 \cdot 45$ & $0 \cdot 274$ & $0 \cdot 609$ \\
$0 \cdot 40$ & $0 \cdot 246$ & $0 \cdot 615$ \\
$0 \cdot 35$ & $0 \cdot 214$ & $0 \cdot 612$ \\
$0 \cdot 30$ & $0 \cdot 1845$ & $0 \cdot 615$ \\
$0 \cdot 25$ & $0 \cdot 152$ & $0 \cdot 608$ \\
$0 \cdot 20$ & $0 \cdot 119$ & $0 \cdot 595$
\end{tabular}

Mean value of $L \div$ volume $=0 \cdot 607$.

Standard deviation of $L \div$ volume $= \pm 0 \cdot 00515= \pm 0 \cdot 85 \%$. 
(the concentration of vitamin A). In other words it is assumed that Beer's law governs the relation of depth of blue colour produced to amount of vitamin A present. Notevarp \& Weedon [1936] have shown this assumption to be valid for extinction coefficients measured on the spectrophotometer, and the results given in Table $I$ prove that the photocolorimeter readings also satisfy this relation to a high precision. The procedure was as follows.

A solution was made of an unsaponifiable residue from cod liver oil and the solution was measured into specially calibrated colorimeter tubes from a microburette graduated in intervals of $0.01 \mathrm{ml}$. Care was taken to wipe the burette tip before each delivery in order to remove unsaponifiable residue deposited there by seepage of the solution past the closed tap and evaporation at the tip. The solution in each tube was made up to $1 \mathrm{ml}$. by addition of pure chloroform from a similar microburette.

The solvent used was dry alcohol-free chloroform which had been distilled 5 days previously, and the reagent was a $20 \%$ solution of antimony trichloride (throughout this paper the concentration of antimony trichloride solutions are given as percentages which signify g. solute in each $100 \mathrm{ml}$. of solution) made up 2 days previously in some of the same batch of chloroform. All readings were taken at $20^{\circ}$. Each reading was taken once only; no duplicates were read. From the figure given for the standard deviation it appears that when working with this degree of care only 1 observation in 22 will err by more than $\pm 1.7 \%$ from the true value of $L$.

These figures show how small is the error of the method, which includes both the error of transferring a measured aliquot of test solution to the colorimeter tube and the personal and instrumental errors in the galvanometer reading. Unless the closest attention is paid to the measurement of the volume of test solution, this degree of accuracy cannot be obtained, as a much larger error will be made in this operation. In any reading this error is likely to control the size of the error of the complete process of taking the reading.

Concentration and purity of the reagent. Fig. 2 shows the effect of varying the concentration of the solution of antimony trichloride. Carr \& Price [1926] originally suggested using a $30 \%$ solution in chloroform. In our experience a

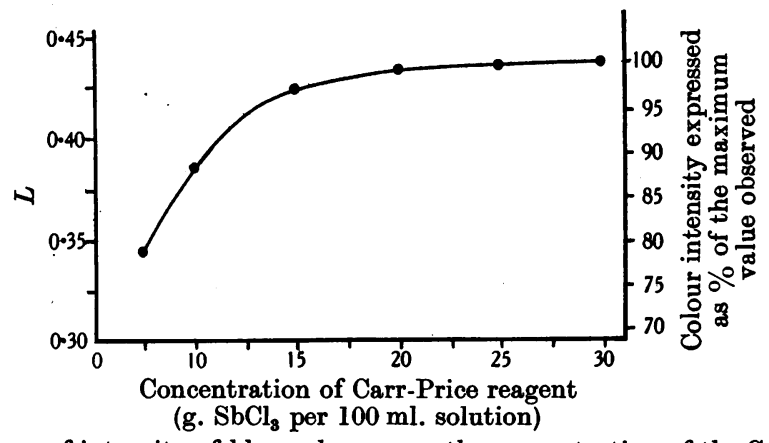

Fig. 2. Dependence of intensity of blue colour upon the concentration of the Carr-Price reagent. Each reading was made by treating $1 \mathrm{ml}$. of a standard solution of vitamin A with $9 \mathrm{ml}$. of Carr-Price reagent of the concentration shown by the individual point.

solution of this concentration can be prepared from reagent quality antimony trichloride and reagent quality chloroform by warming, and the solution will generally remain stable down to $20^{\circ}$ at least; but if the driest commercial specimens of antimony chloride are chosen and dissolved in pure dry alcohol-free 
chloroform at this concentration, then much of the salt will crystallize out at $20^{\circ}$. After the solution and solid have been standing for some weeks, however tightly stoppered (in dark or light), the salt can be redissolved by warming and will not again crystallize out on keeping at $20^{\circ}$. The data charted in Fig. 2 were therefore obtained with a stale reagent.

Fig. 2 shows that there is no need to increase the reagent concentration above $20 \%$, and that the concentration need not be very accurately controlled. This is a great advantage in the use of such a hygroscopic reagent.

Carr \& Price [1926] stated that traces of water and alcohol do not affect it deleteriously. This statement was denied by Notevarp \& Weedon [1936] who found that water, alcohol or degradation products produced by the action of light on the chloroform all affected the depth of blue colour produced. In our experience the former authors are correct, as shown by the following observations. A fresh $20 \%$ solution (solution $\mathrm{A}$ ) of antimony trichloride in pure dry alcohol-free chloroform was prepared. From this solution were prepared a solution B consisting of $99 \mathrm{ml}$. solution $\mathrm{A}+1 \mathrm{ml}$. alcohol; solution $\mathrm{C}, 95 \mathrm{ml}$. of solution $A+5 \mathrm{ml}$. alcohol; and solution $D, 99 \mathrm{ml}$. of solution $A+1 \mathrm{ml}$. water. Solution $\mathrm{D}$ became cloudy, but on standing it separated into two clear layers, the lower very small in volume. Only the upper layer was used for this test. Solution E was a $20 \%$ reagent solution which had been standing in a corked flask in a bright room for 6 months and had separated into large upper and small lower immiscible layers. The upper layer was tested. When each of these solutions was used as reagent with $1 \mathrm{ml}$. of the same solution of vitamin A, triplicate readings were obtained which were identical within the limits of error of the method. Thus for the vitamin concentrations permissible in this method stale reagents or reagents containing traces of water, alcohol or degradation products do not affect the initial depth of the colour produced. This again is very convenient since the reagent solution is hygroscopic and unstable.

Fading of the blue colour. The greatest difficulty in spectrophotometric measurement of the blue colour is due to the rapidity with which the colour

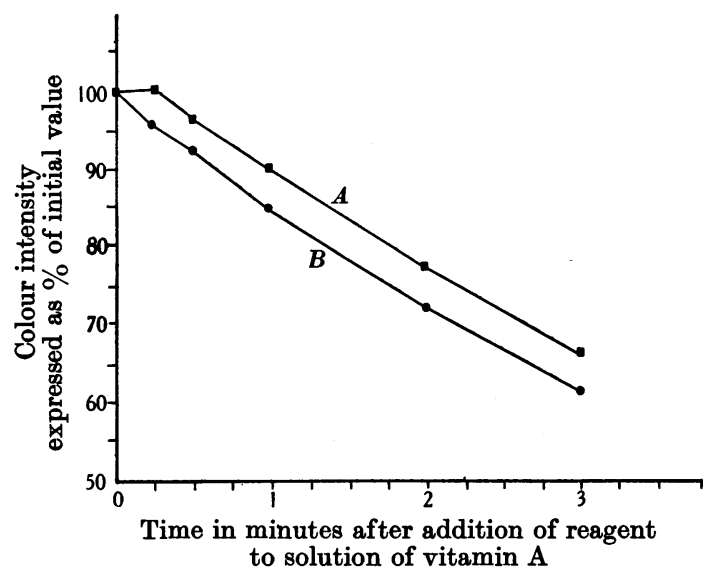

Fig. 3. Observations on the rate of fading of the blue colour. Curve $A$ was obtained by using a freshly prepared reagent solution; curve $B$ with a stale reagent solution. The initial readings with each reagent were identical.

fades. This difficulty is completely overcome by the photoelectric colorimeter, as the reading on any test solution can be taken within 2-3 sec. of adding the 
reagent; this allows time for the mixing of the test and reagent solutions and the swing of the galvanometer spot.

Carr \& Price [1926] claimed that the blue colour is "stable" for 3 min. after its appearance, but our experience shows on the contrary that the colour begins to fade at once if a stale reagent solution is used, and after 10-15 sec. if a fresh solution is used. When a stale reagent solution is added to a test solution in a tube already in position, the galvanometer spot moves rapidly to an initial reading and then immediately begins to drift slowly to the right, indicating a decreasing intensity of colour. But with fresh pure reagent solution the spot moves equally rapidly to the same initial position and then remains motionless for 10-15 sec. before it begins to drift away. Thus there is a real advantage in using the fresh pure reagent solution as the reading can be noted in more leisurely fashion. Moreover, it allows a test to be made of the thoroughness with which mixing occurs in the tube when the reagent is added, as there is time to add the reagent and shake the tube well before placing it in position. This test shows that complete mixing occurs very rapidly when the reagent is added to the tube already in position.

The rate of fading of blue colour produced from one and the same test solution with fresh and with stale reagent solutions is shown in Fig. 3. The rate of fading appears to vary somewhat with the particular test material, but in our experience the difference between the fresh and stale reagents is always as shown in Fig. 3. Similar fading occurs whether the test solution contains an oil or an unsaponifiable residue.

Effect of temperature. We have confirmed earlier observations that temperature exerts an effect on the depth of colour produced, but no accurate measurements have been possible. Readings were taken in a warm room, and the instrument and solutions transferred to a refrigerator where further readings were taken after some hours. Measurements were first made at $21^{\circ}$ and $11^{\circ}$ respectively, and the $L$ value was found to be $4.8 \%$ greater at the lower temperature. Later another test was made at $29^{\circ}$ and $5^{\circ}$ and $L$ was observed to be $10.6 \%$ greater at the lower temperature. From these figures it seems that the observed value of $L$ is increased by about $4-5 \%$ for a temperature decrease of $10^{\circ}$ within the range $0-30^{\circ}$ : this is much less than was claimed by Wokes \& Willimott [1927], but a careful reading of their paper shows that there was some confusion between depth of colour produced and the rate at which it developed.

The chromogenic power of carotene relative to its yellow colour. The following observations were made in order to calculate the numerical factor employed within the bracket of equation (3) above. $23 \cdot 3 \mathrm{mg}$. of commercial $\beta$-carotene were weighed from a freshly opened ampoule and dissolved in $10 \mathrm{ml}$. of chloroform. Further dilutions were made and $L_{440}$ for each of the resultant solutions was measured. It was found that the solutions obeyed Beer's law with precision and the $L_{440}$ value of the $\beta$-carotene was 1440 . A series of lesser dilutions was also prepared and to $1 \mathrm{ml}$. of each solution $9 \mathrm{ml}$. of the $20 \%$ antimony trichloride were added and $L_{620}$ measured for each. It was found that the blue solution obeyed Beer's law, and the $L_{620}$ value of the carotene was 220 .

The ratio of $\frac{L_{620}}{L_{440}}$ was $\frac{220}{1440}$ or $0 \cdot 153$.

Similarly with a commercial specimen of carotene stated to contain $25 \%$ of $\alpha$-carotene the values observed were $L_{440}=1641, L_{620}=220$ and $\frac{L_{620}}{L_{440}}=0 \cdot 130$. The absolute values of $L$ recorded here are undoubtedly too low on account of the difficulty of obtaining pure specimens of carotene; we hope to be able to prepare pure specimens and redetermine the figures in the future. The ratios are likely 
to be more accurate, and for the present the mean value of the two determinations is suggested for use in equation (3).

Relation of $L$ value to the other units. Since it was felt that the present method has materially increased the precision and accuracy of the measurement of the blue vitamin A-antimony trichloride complex, it appeared desirable to determine experimentally the degree of correlation between the new $L$ value, the $E$ value at $328 \mathrm{~m} \mu$ and the blue value. For this purpose a series of measurements was made on unsaponifiable residues (except in the case of concentrated sources) to eliminate factors of inhibition which constitute an important (though entirely unnecessary) source of discrepancy between the chemical and physical values. The conversion factors for the above units were determined on the basis of measurements on 21 oils and 18 concentrates as follows:

$E(1 \%, 1 \mathrm{~cm} ., 328 \mathrm{~m} \mu)=L$ value $\times(0 \cdot 41 \pm 0 \cdot 05)$.

Blue value $=L$ value $\times(20 \pm 3 \cdot 0)$.

The ratio of $L$ value to $E$ value at $620 \mathrm{~m} \mu$ was calculated from equation (1) and was not measured on these oils and concentrates. The calculation showed that $E(1 \%, 1 \mathrm{~cm} ., 620 \mathrm{~m} \mu)=L$ value $\times(1.30 \pm 0.03)$. Bearing in mind the nature and magnitude of the various sources of discrepancy so ably discussed by Morgan et al. [1935], we feel that the above conversion factors will yield accurate results (within the limits indicated above) for those who wish to change from one unit to another. The question of the correlation of the $L$ value with the biological assay will be dealt with separately.

\section{SUMMARY}

1. The Evelyn photoelectric colorimeter has been applied to the measurement of the intensity of the blue colour produced by the reaction between vitamin $A$ and antimony trichloride; the simple procedure for the estimation of vitamin $A$ in oils and other materials is described. The method combines accuracy, convenience and economy perhaps to a greater extent than any other.

2. The most important advantage of this new method is its objectivity which ensures that the results obtained are as far as possible independent of the operator, thereby eliminating a source of serious error. Other advantages are rapidity and convenience.

3. Data are presented to illustrate the precision of the method, which is considerably greater than that obtained with the tintometer or the visual spectrophotometer.

4. A new unit is defined for the expression of results obtained by the method, and experimental data are given on the relation between the new unit and other units now in use.

One of us (K. A. E.) is indebted to the Banting Research Foundation, Toronto, Canada, for several grants during the period of development of the colorimeter as well as in support of the present work. 


\section{REFERENCES}

Carr \& Price (1926). Biochem. J. 20, 497.

Coward, Dyer, Morton \& Gaddum (1931). Biochem. J. 25, 1102.

Drummond \& Morton (1929). Biochem. J. 23, 785.

Emmerie (1933). Nature, Lond., 131, 364.

Evelyn (1936). J. biol. Chem. 115, 63.

Holmes \& Corbet (1937). J. Amer. chem. Soc. 59, 2042.

Hume (1937). Nature, Lond., 139, 467.

McFarlan, Reddie \& Merrill (1937). Ind. Eng. Chem. Analytical Ed. 9, 324.

Medical Research Council (1935). "The Standardisation and Estimation of Vitamin A." (H.M. Stationery Office, London.)

Morgan, Edisbury \& Morton (1935). Biochem. J. 29, 1645.

Notevarp \& Weedon (1936). Biochem. J. 30, 1705.

Takahashi, Nakamiya, Kawakami \& Kitasato (1925). Proc. Tokyo Inst. Phys. and Chem. Res. $3,81$.

Wokes \& Willimott (1927). Analyst, 52, 515. 